\title{
A three stage segmentation method of kidney and kidney tumor
}

\author{
Zhan Wei \\ HangZhouDianZi University, China
}

\begin{abstract}
The morphometry of a kidney tumor revealed by contrastenhanced Computed Tomography (CT) imaging is an important factor in clinical decision making surrounding the lesion's diagnosis and treatment. I Use 3D Unet to segment kidney and kidney tumor respectively.
\end{abstract}

\section{First Stage}

The purpose of first stage is to segment kidney roughly

\subsection{Preprocessing}

For the preprocessing in the First stage, I adjust all the CT Value to [-200,300] to remove irrelevant information, and resample all the data to $3 \mathrm{~mm} * 3 \mathrm{~mm} * 3 \mathrm{~mm}$, padding all the data to $256 * 192 * 192$ so that all the data can be put into the model, than all the data was normalized instancely

\subsection{Architecture}

3D Unet was used for the first stage

\subsection{Training details}

The learning rate was initialized as $3 \mathrm{e}-4$, and multiplied by 0.3 after every 50 epoches. I use Adam optimizer. For loss function, bce+dice loss was used. Total epoch was set to 150 , It takes about 14 hours to train a model

\subsection{Post processing}

For the first stage, I do not use any post processing

\section{Second Stage}

The purpose of the second stage is to segment the kidney accurately

adfa, p. 1, 2011.

(C) Springer-Verlag Berlin Heidelberg 2011 


\subsection{Preprocessing}

For the preprocessing in the second stage, I adjust all the CT Value to [-200,300] to remove irrelevant information, then random cropping, contrast augmentation, brightness augmentation, random flipping by $w$ axis, gamma augmentation, random rorate the image were implemented to all the training data, and resample all the data to $1.5 \mathrm{~mm} * 0.8 \mathrm{~mm} * 0.8 \mathrm{~mm}$, then padding them to $144 * 224 * 432$ so that all the data can be put into the model, than all the data was normalized instancely.

\subsection{Architecture}

3D Unet was implemented for the second stage

\subsection{Training details}

The learning rate was initialized as $3 \mathrm{e}-4$, and multiplied by 0.3 after every 50 epoches. I use Adam optimizer. For loss function, bce+dice loss was used. The total epoch was set to 150 , It takes about 14 hours to train a model

\section{$2.4 \quad$ Post processing}

For the post processing, I get the maximum connected component of the output of trained model, then, all the connected whose voxels more than $0.1 *$ voxels of maximum connected component are kept, and all the connected component whose voxels less than $0.1 *$ voxels of maximum connected component are dropped.

\section{Third Stage}

The purpose of the third stage is to segment the kidney tumor accurately. The Preprocessing, architecture, training details and post processing is as same as that in the second stage. 